\title{
Nurses' knowledge towards severe acute malnutrition management protocol and its associated factors
}

\author{
Abdu Oumer \\ Department of Public Health, Wolkite University, Ethiopia
}

\begin{tabular}{l}
\hline Article Info \\
\hline Article history: \\
Received Mar 13, 2019 \\
Revised Apr 17, 2019 \\
Accepted May 11, 2019 \\
\hline
\end{tabular}

\section{Keywords:}

Knowledge

Management

Nurses

Severe Acute Malnutrition

Children

\begin{abstract}
For appropriate management of severe acute malnutrition skilled, knowledgeable and concerned health professionals are critical for child survival. Thus assessing the knowledge of nurses towards management protocol of severe acute malnutrition is crucial step for targeted interventions. This study aimed to assess Knowledge towards Severe Acute Malnutrition Management Protocol and its Associated Factors among Nurses working in Hiwot Fana Specialized University Hospital, 2018. Cross-sectional study was conducted among eligible 132 nurses. Data were collected using self-administered questionnaire prepared from the national SAM management guideline of Ethiopia. SPSS version 20.0 software using frequency, tables, graphs, percentages and mean was used. Student T test and analysis of variance with $F$ statistics and $P$ value was computed. Overall, $65(49.2 \%)$ of nurses had poor knowledge on SAM management. More than half, $100(75.8 \%)$ of the nurses had experience in SAM management previously. Males were more likely to be knowledgeable (AOR=1.27) as compared to females. Nurses with the previous experience of managing malnourished child had 1.70) times more likely to be knowledgeable as compared to their counterparts. Having SAM training was associated with having higher knowledge score (AOR=1.56). Having SAM training was found to have significantly higher knowledge score $(\mathrm{p}=0.034)$. Knowledge level of nurses towards SAM management is not satisfactory. Those who ever involved in SAM management, having recent malnutrition training and gender were predictors of high knowledge score. There should be regular capacity building schemes for nurses especially for those who are involved in management of SAM at emergency or SAM unit.
\end{abstract}

Copyright () 2019 Institute of Advanced Engineering and Science. All rights reserved.

\section{Corresponding Author:}

Abdu Oumer,

Lecturer, Department of Public Health,

Wolkite University, Ethiopia.

Email:phnabu@gmail.com

\section{INTRODUCTION}

Severe Acute Malnutrition (SAM) is defined as very low weight for height (below-3 z scores or below 70\%), visible severe wasting, or the presence of nutritional edema or mid upper arm circumference (MUAC) below $11.5 \mathrm{~cm} \mathrm{[1]} \mathrm{or} \mathrm{MUAC} \mathrm{below} 11 \mathrm{~cm}$ in Ethiopia [2]. It results from sudden period of food shortage and is associated with a loss of body fat and wasting of muscle mass. Clinically it present in three forms namely marasmus, kwashiorkor or marasmic kwashiorkor [3]. Currently around 52 million children are wasted globally with estimated magnitude of SAM 19 million. In Africa about $7 \%$ of children are wasted [4], which far from 2025 target to achieve wasting below 5\% [5]. As of the 2016 estimate, globally 7.5\% of children suffered from wasting [4], which is far from the Sustainable Development goal of below 5\% [5]. It is affirmed that, among the nutritional deficiency indicators wasting has shown a slow progress in Africa and Asia where about 28\% of wasting is found [6]. 
As the physiological system of malnourished children is significantly reduced by the principle of reductive adaptation, which results in altered response to medications and others. Thus the consequences of maltreatment is greater than the natural course [1]. Similarly as the principles of treatment of well-nourished child is different from malnourished, successful treatment of such cases need strict consideration of the metabolic capacity of the child like fluid therapy which are critical. SAM significantly affects child survival, countries economic productivity, long-term cognitive decline and other negative health [6] consequences. Successful management of the severely malnourished patients requires that both medical and social problems be recognized and corrected [7]. For effective and successful management, it requires that each child be treated with proper care and affection in addition to nutritional therapy (F75, F100 and RUTF) and treatment of Medical complications [2]. Considering the above phenomena, the WHO and UNICEF adopted cost effective approach to address acute malnutrition, that community based management of acute malnutrition in which impatient therapy for complicated SAM children is one option [8].

As the management is different from well-nourished children in the protocol, in appropriate management is common among non-trained individuals. For example reported from Mali showed that only $79.5 \%$ of cases were correctly assessed, classified and treated and correctly managed by nurses. While significant number of children were not treated according to the guideline, in terms of antibiotics, nutritional therapy and above all fluid related treatment for severe dehydration and shock [9]. This may have great impact on the current burden of SAM on underfive mortality and child survival [3].

SAM causes significant number of mortality among malnourished children. But with the correct implementation of the national SAM guideline using the ten principles of SAM treatment can reduce mortality and improve recovery. In Ethiopian context where CMAM is implemented with four target oriented programs, Nurse Professionals are the major contributors of SAM management stating from screening to impatient SAM management. Especially in specialized health facilities where impatient facility is established and treatment of complicated cases of malnutrition are treated health professional knowledge on SAM management protocol is essential to ensure adherence to the appropriate treatment [3]. Implementation integrated and high quality SAM management care in line with the protocol shown to improve child survival and with moderate recovery rate [10].

Even if there many facility related factors that pose significant risk on low treatment effectiveness among SAM children, lack of appropriate skills and training of health professional pose significant negative influences [11]. As the treatment of SAM is integrated management of both nutritional deficiency and medical co morbidities, health professional need to be aware and cable to give appropriate care for SAM children [12]. With the existing significant effort, there is significant decline in mortality from SAM 55\% to below $20 \%$, but the mortality rate attributable to various causes is still high [3]. Still the mortality among this children reaches up to $46 \%$ in other countries [13-14] and about 29\% in North West Ethiopia [15]. Other studies from Northern Ethiopia and Eastern part showed mortality rate of $12.8 \%$ [16] and 9\% [17] respectively where majority of deaths occurs the first weeks of therapy where many medical complications are maltreated. Additionally the sphere project minimum standard also sets the death rate below $10 \%$, default rate of $15 \%$ and the nutritional recovery rate of $75 \%$ [18]. Among this in appropriate implementation of SAM protocol in particular setting by health care providers is of significant value.

This study tried to assess the knowledge and perceptions of nurses towards national management protocols of for children 2016 version. It will give a valuable information on any gaps in skills and training of health professionals more specifically on malnutrition. This allows the hospital and regional Health bureau for appropriate programs and training modalities to increase the nurse's technical knowledge and skills in the management of malnutrition in accordance to the national SAM management protocol.

\section{Objectives of the study}

To assess correlates of nurse's knowledge towards SAM management protocol among in Hiwot Fana Specialized University Hospital (HFSUH), Eastern Ethiopia, 2018.

\section{METHOD}

\subsection{Study setting and design}

The study was conducted in HFSUH in Harari town. The region has about five hospitals and numerous health centres and Health posts delivery comprehensive primary health care to the community. The city is located $526 \mathrm{Km}$ from the capital Addis Ababa. It has two government Hospitals, Federal Police Hospital, two private Hospitals, eight Health Centers and many private clinics serving the people of the state. HFSUH is one of the two government Hospitals with a total of 1,000 staffs among these 600 of them are health care professionals and 200 are nurses. The hospital, apart from giving daily different medical services 
including management of different forms of malnutrition including SAM. Institutional based Cross sectional study were used to assess nurses` knowledge on SAM management protocol in 2018.

\subsection{Populations and eligibility criteria}

All nurse professionals in HFSUH that are physically present during the data collection period were included in the survey while all nurses in HFSUH (estimated 200) were the source population. Those who were in annual leave at the time of data collection, sick and were not on job during this period were excluded.

\subsection{Variables of the study}

Knowledge of Nurses` towards SAM Management Protocol was the dependent variable of the study while sex, Age, working experience, curriculum, qualification, Unit of work were the main predictor variables related to their knowledge level.

\subsection{Sample size determination and sampling technique}

Sample size was computed based on single population proportion formula by taking the knowledge level of health professionals towards SAM management protocol (50\%), at $95 \%$ Confidence level and 5\% margin of Error (d), standard critical value (za/2) of 1.96 at $95 \%$ confidence level, the sample size became 384. But the total number of nurses in the Hospital were estimated to be a total of 200, which is less than the sample size calculated (384). As the sample size calculated is greater than $5 \%$ of the total population, finite population was used to calculate the effective final sample with $10 \%$ non-response rate, the final sample size was 145 . Since the actual sample and the expected total population are almost equal, all efforts were tried to include all nurses during the data collection period (the effective sample size was 132).

\subsection{Data collection methods}

The data collection instrument was pre-tested before the data collection. An anonymous self-administered questionnaire was adopted and modified after reviewing different literature mainly the components of national SAM management protocol in English language [2]. Trained data collectors were used to explain objective of the study, collect the filled questionnaire and give guidance for the participants.

\subsection{Data quality management and data analysis}

Pre-testing was conducted in Haramaya hospital prior to data collection process. Based on the pre-test, questions were revised and edited with necessary modification. Questionnaires were prepared in English since the study populations were educated and can read \& understand the concept of the questions this was minimize the risk related with questioner translation. Data was analyzed using SPSS version 20 software package. The data was cross checked prior to actual analysis. The data were presented using mean, standard deviation, percentage, graphs and tables. Additionally one way analysis of variance was done to compare the mean knowledge score of the study subjects.

\subsection{Ethical considerations}

Ethical clearance was obtained from institutional Ethical review committee of Harar Health Science College before the staring of the field work. Respondents were informed about the objective and purpose of the study and verbal consent was obtained from each respondent. Moreover, all the study participants were informed that they have a full right to participate or decline from participating in the study and the study participants were assured for an attainment of confidentiality for the information obtained from them.

\subsection{Operational definitions}

Good Knowledge: when overall knowledge score of study participant's is above mean. All correct answers were coded as 1 while the incorrect one were coded as 0 , then the sum and mean of the sum of observations was calculated to define the knowledge cut off point. Similarly poor knowledge was defined as overall knowledge score below the mean knowledge score of the sample [19].

\section{RESULTS AND DISCUSSION}

\subsection{Socio demographic characteristics}

A total of 132 nurses were included in this study. Out of this $75(57 \%)$ were females working in different units of the Hospital. Majority (42.4\%) were in the age below 30 years. The mean age of the nurses were 32.2 years $(32.2$ years \pm 5 years). Only few, $11(8.3 \%)$ has less than one years working experience, while $63(47.7 \%)$ had 1-5 years working experience, $40(30.3 \%)$ has 6-10 years working experience and about $13 \%$ had work experience above ten years it is presented in Table 1. 
Table 1. Socio demographic characteristics of nurses

\begin{tabular}{cccc}
\hline Variables & & Frequency & Percent $(\%)$ \\
\hline \multirow{4}{*}{ Sex } & Male & 75 & 57 \\
& Female & 57 & 43 \\
\multirow{4}{*}{ Age } & 19-28 years & 56 & 42.4 \\
& 29-38 years & 45 & 34.1 \\
& 39-48 years & 23 & 17.4 \\
\multirow{5}{*}{ Work Experiences } & 49-58 years & 8 & 6.1 \\
& $<1$ year & 11 & 8.3 \\
& 1-5 years & 63 & 47.7 \\
& 6-10 years & 40 & 30.3 \\
& Above ten years & 18 & 13.7 \\
\hline \multirow{6}{*}{} & & & \\
\hline
\end{tabular}

Among 132 study participants, 39 (29.5\%) were used WHO guideline to treat SAM, 27 (20.5\%) used United Nation International Children Education Fund (UNICEF) guidelines to treat SAM. In addition, only 39 (29.5\%) reported to use Ethiopian SAM management protocols while, 27 (20.5\%) did not used all. Majority of them, $108(81.8 \%)$ nurses covered acute malnutrition in their academic stay while, more than half, $59(44.7 \%)$ covered in their second year of study. A total of $101(76.5 \%)$ of nurses were ever involved in management of SAM. Also, $117(88.6 \%)$ of nurses perceive that the time devoted to childhood severe acute malnutrition section was adequate (Table 2). Majority of the nurses, 98 (74.2\%) had received training on Severe Acute Malnutrition, and interestingly SAM displays (posters) are displayed at the various work place on pediatrics, inpatient and outpatient wards or consultation room, it is presented in Table 2.

Table 2. SAM management related behaviors among nurses

\begin{tabular}{cccc}
\hline Variables & & Frequency & Percent (\%) \\
\hline Was acute malnutrition /Nutrition/ covered & Yes & 108 & 81.8 \\
in your curriculum /previously/? & No & 24 & 18.2 \\
& First year & 26 & 19.7 \\
In which year of study, it is addressed? & Second year & 59 & 44.7 \\
& Third Year & 40 & 30.3 \\
Involvement in management of severe & Fourth Year & 7 & 5.3 \\
acute malnutrition. & Yes & 101 & $76.5 \%$ \\
After being employed have you ever & No & 31 & $23.5 \%$ \\
attended in-service or out-service & Yes & 98 & 74.2 \\
training/s on SAM? & No & 34 & 25.8 \\
\hline
\end{tabular}

\subsection{Nurses` knowledge on SAM management}

About half of nurses, $49.2 \%$ correctly identified the correct MUAC cut off point (less than $11 \mathrm{~cm}$ ) for diagnosing SAM. Regarding antibiotics treatment recommendation for SAM children, $63(47.7 \%)$ said that routine antibiotics should be given for all children. An estimated, 50.8\% and $47.7 \%$ correctly identified the boosted Vitamin A dosage foe less than six months (50,000 IU) and above one year (200,000 IU) respectively. Regarding correct discharge criteria in accordance with the national recommendations, half of nurses correctly identified has no edema and reached target weight correctly. More importantly on the type of electrolyte to be given for SAM with edema as potassium (53\%). While $64.4 \%$ of nurse identified wasting as low weight for height tor length depending on age and $62.9 \%$ of nurses identified WFH/L percentage above $85 \%$ as discharge criteria for older children with SAM, it is presented in Table 3.

In line with routine medication and supplements, majority of them, 61 (46.2\%) said iron can be given at the beginning of Transition phase, 35 (26.5\%) were given iron at stabilization phase while the rest occurred during discharge. Almost three fourth, 100 (75.8\%) of the nurses have ever worked in SAM management in the hospital or elsewhere. Regarding the types of guideline used, 39\% used WHO SAM guideline followed by 35 (26.5\%) used Ethiopian national SAM guideline for management of SAM children, it is presented in Figure 3.

Int. J. Public Health Sci. Vol. 8, No. 2, June 2019: 219 - 228 
Table 3. Some important dimensions of nurses` knowledge on SAM management protocol

\begin{tabular}{|c|c|}
\hline Variables & $\begin{array}{l}\text { Answered correctly } \\
\mathrm{N}(\%)\end{array}$ \\
\hline MUAC cut off to diagnose SAM above six month (less than $11 \mathrm{~cm}$ ) & $65(49.2 \%)$ \\
\hline $\begin{array}{l}\text { Routine Antibiotic recommendation } \\
\text { For all SAM children }\end{array}$ & $63(47.7 \%)$ \\
\hline vitamin A dosage booster for less than six months of $50,000 \mathrm{IU}$ & $67(50.8 \%)$ \\
\hline vitamin A dosage booster for $12-59$ months of age $200,000 \mathrm{IU}$ & $63(47.7 \%)$ \\
\hline Necessary investigation Prior to discharge HIV & $43(32.6 \%)$ \\
\hline $\begin{array}{c}\text { Which one are the Discharge Criteria for SAM Children from 6month to } \\
\text { adulthood? Has no oedema and reached target weight }\end{array}$ & $66(50 \%)$ \\
\hline $\begin{array}{l}\text { Correctly state discharge Criteria for Children }<6 \text { month or }<3 \mathrm{~kg} \text { being breast } \\
\text { fed Weight gain on breast milk alone Has no medical problem }\end{array}$ & $63(47.7 \%)$ \\
\hline Appropriate electrolyte for child having worsening edema? Potassium & $70(53 \%)$ \\
\hline How is wasting defined in the road to health booklet? Low weight for height & $85(64.4 \%)$ \\
\hline $\begin{array}{l}\text { Not used as discharge criteria for a child with SAM The height for age is on } \\
\text { the 0-line target }\end{array}$ & $43(32.6 \%)$ \\
\hline $\begin{array}{c}\text { Which one is the Discharge Criteria for Children aged } 6 \text { month to } 59 \text { months } \\
\text { old? W/L or WFH }>85 \% \text { or target weight gain achieved }\end{array}$ & $83(62.9 \%)$ \\
\hline
\end{tabular}

Regarding nurses` knowledge on SAM management protocol, out of the 23 questions 65 (49.2\%) had poor knowledge (scored below the mean) while, 67 (50.8\%) had good knowledge on malnutrition and its managements (had answered above the average questions correctly). Out of the total 100 points, the mean knowledge score of nurses was above the average, 53.9 with standard deviation of 14.4 points. Almost half, $65(49.2 \%)$ of nurses mentioned all types of nutritional therapies as necessary for management of severe acute malnutrition children. While, $32(24.2 \%), 18(13.6 \%)$ and $17(12.9 \%)$ of nurses correctly stated F-75 milk, F-100 milk and RUTF as appropriate nutritional supplement, it is presented in Figure 1.

About $50(37.9 \%)$ were reported most of death related to malnutrition are caused by dehydration, Anemia and shock. While $43(32.6 \%)$ dictated that majority of SAM related deaths are caused by hypocalcaemia, infection and dehydration. Similarly respondents stated that, $32(24.2 \%)$ of respondents stated that electrolyte imbalance and hypoglycemia and hypothermia are predominant causes SAM related deaths. Regarding fluid therapy, 18 (13\%) of nurses recommend fluid therapy by intravenous route for SAM children as opposed to oral route. With respect to diuretic therapy, all did not recommend antidiuretic therapy for SAM child with edema, it is presented in Table 3 and Figure 2.

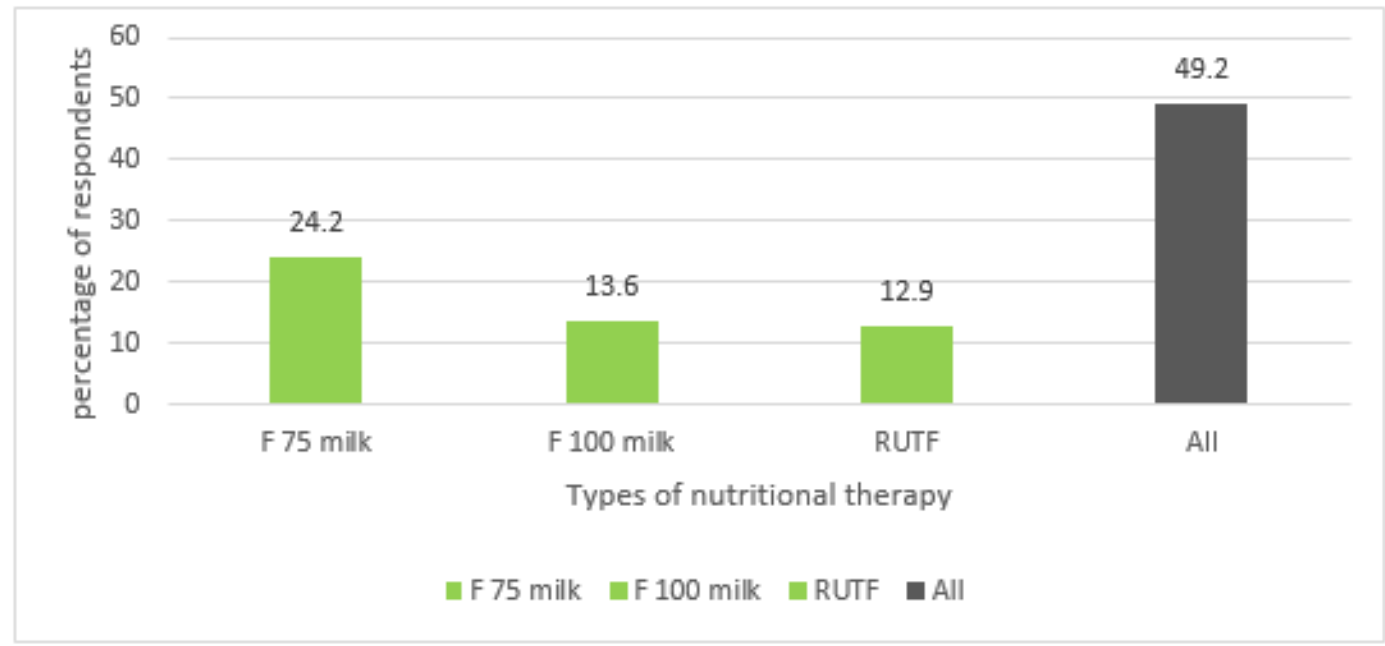

Figure 1. Perceived type of nutritional therapy given for children among nurses 


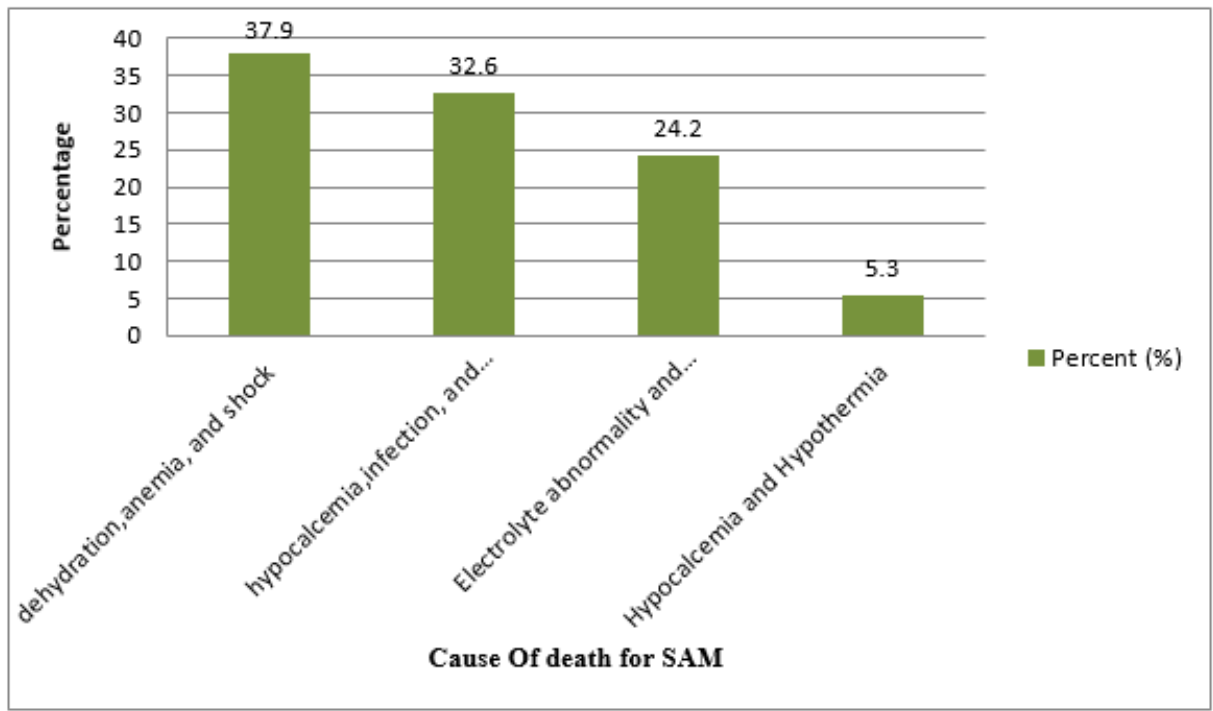

Figure 2. Perceived causes of death in children with severe acute malnutrition

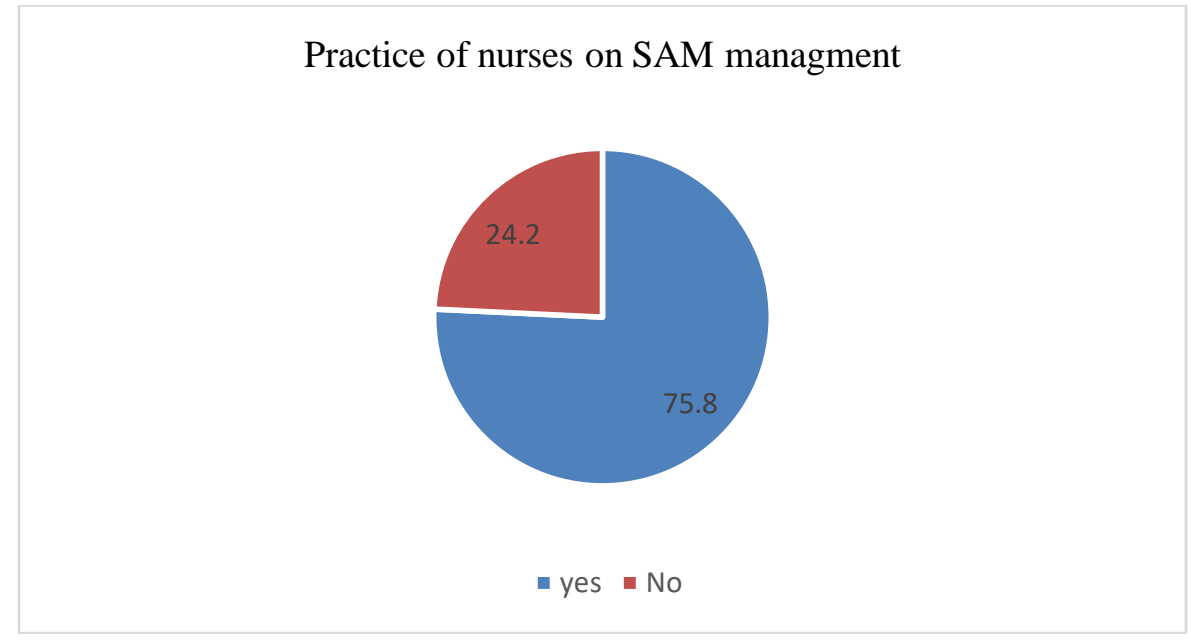

Figure 3. Severe acute malnutrition management practices among nurses

\subsection{Correlates of knowledge on SAM management}

Being ever involved in SAM management, having recent SAM training and gender were the major factors associated with knowledge level of nurses $(p>0.05)$. Male nurses were more likely to be knowledgeable/aware of/SAM for children ( $\mathrm{AOR}=1.27(95 \% \mathrm{CI}=0.62-2.60)$ ) as compared to females. Nurses with the previous experience of managing malnourished child had 1.70 (95\% $\mathrm{CI}=0.72-4.04)$ times more likely to be knowledgeable as compared to their counterparts. They are who having SAM training has shown positive influence on the knowledge score of Nurses on SAM management protocol (AOR=1.56, 95\% $\mathrm{CI}=(0.67-3.61)$. Table 4 shows that information in detail.

Since the number of groups of predictors were two categories, thus the analysis of variance is used instead of student $t$ test as the results of both is the same and the estimated type I error $(\alpha)$ remain the same. Nurses having SAM training was found to have significantly higher knowledge score than without training $(\mathrm{p}=0.034)$. While those with previous experience of managing SAM children had higher knowledge score than the counters $(\mathrm{p}=0.034)$. Those data is presented in Table 5.

This particular study assessed the knowledge and its correlates among nurses in Hospital, Eastern Ethiopia. The finding of the study showed that, 65 (49.2\%, 95\% CI=40.7\% to 57.4\%) had poor 
knowledge on malnutrition and its management. Males nurses $(\mathrm{AOR}=1.27(95 \% \mathrm{CI}=0.62-2.60))$, those with previous experience on managing malnourished child $(\mathrm{AOR}=1.70(95 \% \mathrm{CI}=0.72-4.04)$ ) and having $\mathrm{SAM}$ training $(\mathrm{AOR}=1.56,95 \% \mathrm{CI}=0.67-3.61)$ was positively associated with higher knowledge score. The knowledge level of nurses was not adequate and satisfactory. Similar study also showed the level of nurses knowledge was not universal, which showed greater level of knowledge among nurses of 74\% [20]. On the other hand, the study [21] did not report an overall knowledge score but did conclude that nurses had poor knowledge about management of malnutrition in children. In addition, about 54\% [22] of nurses were knowledgeable regarding malnutrition and its management. Similarly study conducted among nurses showed lower less than average knowledge score (mean=49 points with SD of 10) [23]. According to the WHO updated SAM guideline (2013) insufficient knowledge amongst nurses can lead to an incorrect diagnosis being made which could bring poor clinical outcomes for the patient [1]. Similarly, same study conducted among health care workers reported that the overall knowledge level of nurses was poor. In addition, they lack basic skills in assessing and managing SAM appropriately [24].

Table 4. Factors associated with knowledge level of nurses on SAM management

\begin{tabular}{cccccc}
\hline \multirow{2}{*}{ Variables } & \multirow{2}{*}{ options } & Knowledgeable & Not knowledgeable & AOR & p value \\
\hline \multirow{2}{*}{ Gender } & Female & 35 & 40 & 1 & \\
& Male & 32 & 25 & $1.27(0.62-2.60)$ & 0.520 \\
Ever involved in & Yes & 53 & 50 & $1.70(0.72-4.04)$ & 0.228 \\
SAM management & No & 14 & 15 & 1 & \\
& Yes & 53 & 43 & $1.56(0.67-3.61)$ & 0.301 \\
SAM training & No & 14 & 22 & 1 & \\
& & & & & \\
\hline
\end{tabular}

Table 5. Mean comparison of knowledge score versus training and experience in managing SAM children.

\begin{tabular}{ccccccc}
\hline & Analysis of Variance table & & & & \\
& & Sum of & Dean & F & Sig. \\
& & Squares & Dfuare & F & & \\
\hline knowledge score out of & Between Groups combined & 584.440 & 1 & 584.440 & \multirow{2}{*}{4.617} & \multirow{2}{*}{$0.034^{*}$} \\
$100 \% *$ Training on SAM & Within Groups & 16457.570 & 130 & 126.597 & & \\
Knowledge score *ever & Between Groups combined & 372.122 & 1 & 372.122 & 2.902 & .091 \\
involve in SAM management & Within Groups & 16669.887 & 130 & 128.230 & & \\
\hline
\end{tabular}

* df refers to degree of freedom

This study showed having training on the SAM and its management significantly predicted the knowledge level of Nurses. Thus training is an important tool to improve the Nurses' knowledge, perception and skills. On the other side, the knowledge of nurses towards SAM management were not adequate [21, 24], indicates the need for evidence based interventions to address high hospital mortality among SAM children. It is also evident that, reductive adaptation that occurs in SAM children makes more vulnerable to complications related to maltreatment. Especially excessive fluid therapy, treatment related to dehydration and electrolyte disturbance make them susceptible to develop complication and early treatment related deaths, which needs critical and concerned skills to be acquired by professionals [1-2].

With the implementation of SAM management protocol, substantial reduction in mortality from $55 \%$ to below $20 \%$ is observed, but still the case fatality rate is unacceptably high [3, 17]. In country like Ethiopia also $6 \%$ to $29 \%$ mortality has been observed among admitted SAM children is reported [15, 25]. It is also evident that misdiagnosis ad mistreatment by health care professional due to lack of knowledge and skill contribute to large case fatality rate [26].

This emphasize, the need for health professional who are involved in management of SAM to strictly adhere themselves to the updated national SAM management protocol with aim to have optimal treatment outcome in terms of death and recovery [27]. So, regular training of nurses on nutritional screening and SAM management protocol with subsequent refreshment training is of valuable importance in order to early detect and appropriately treat SAM children. In support of the need for training study on health 
professionals, since nurses' knowledge on SAM screening and management was less than 30\% [24]. Additionally having previous experience in screening malnourished children scored higher knowledge score than counter parts [23]. Which is similar with our finding, emphasizing the need for experience in order to develop skills and psychomotor development among nurses for better care of SAM children than theoretical knowledge.

Similar study showed that, having refreshment training increase the knowledge level of nurses on SAM and its managements [22]. Even if, it is not significant, nutrition course coverage should strictly address the major public health concerns in colleges and universities like malnutrition, SAM management protocol and nutritional assessments. This will help nurses to help build their capacity in malnutrition and its management. Additionally lack of continuity in training and refreshment training had significant contribution on management of SAM among health care providers in Africa [26], in that manuals and clinical evidence may be updated from time to time.

Similar findings also showed that, critical knowledge gaps were also noted regarding areas that focused on the clinical nutritional management of severely malnourished children. About $15 \%$ of the nurses knew that high-protein diets should not be prescribed for children presenting with kwashiorkor. Also, about $70 \%$ of them did not know that standard ORS should not be used to treat or prevent dehydration in SAM children [28]. This might be attributed to low access to regular training and refreshment workshops on malnutrition for health professionals including nurses.

This can be regarded as basic information and a score of $100 \%$ would have been expected. But knowing the critical aspects of fluid therapy, nutritional supplement, micronutrient supplement and other had great impact on treatment outcome (Mortality). This is of critical importance to reduce mortality risk, relapse and increasing recovery [2]. This is important to achieve the global target to reduce acute malnutrition below 5\% which is not achieved yet [29], but it causes an estimated one million of under-five deaths and its long term physical, cognitive and productivity losses in long term

\section{CONCLUSION}

Generally the knowledge level of nurses towards SAM management is not satisfactory. Those who ever involved in SAM management, having recent malnutrition training and gender were predictors of high knowledge score. This knowledge aspect is critical to achieve high recovery, reduced mortality and reduced relapse of malnutrition.

In general there should be regular capacity building schemes for nurses especially for those who are involved in management of SAM at emergency or SAM unit. So HFSUH in collaboration with other stakeholders should plan, facilitate and organize in service training for nurses focusing on nutritional assessment, malnutrition and malnutrition management. In addition, malnutrition charts and other facilities should be in place that nurses can access.

\section{ACKNOWLEDGEMENTS}

Many thanks to study participants (HFSUH Nurses) for their time and valuable information and data collectors (Pharmacy students) for their contributions.

\section{REFERENCES}

[1] World Health Organization (WHO), Update on Management of Severe acute malnutrition in infants and childrens, in Guideline2013, World Health organization: Geneva. p. 14-46.

[2] FMOH, Training Course On the management of severe Acute malnutrition, Ministry Of Health, 2013, FMOH: Addis Ababa, Ethiopia. p. 10-72.

[3] Bhutta, Z., et al., Evidence-based interventions for improvement of maternal and child nutrition: what can be done and at what cost? Maternal and Child Nutrition, 2013. 2: p. 1-15.

[4] Global Nutrition Report (GNR), Global Nutrition Report Action and Global Nutrition Report: Current Global Estimates, 2016. P. 20-46.

[5] Endang, et al., Global Nutrition Report Action and accountablity in Global Nutrition Report 2014. p. 20-40. 
[6] World Health Organization (WHO), Maternal, infant and young child nutrition in East and Southern African countries: moving to national implementation, in Report of a World Health Organization workshop, Worldhealthorganization, Editor 2014, Geneva, WHO: Entebbe, Uganda. p. 7-12.

[7] Trehan, I., et al., Antibiotics as Part of the Management of Severe Acute Malnutrition. N Engl J Med 2013. 368: p. 425-35.

[8] Tekeste, A., et al., Cost effectiveness of community-based and in-patient therapeutic feeding programs to treat severe acute malnutrition in Ethiopia. Cost Eff Resour Alloc, 2012. 10(4): p. 1-10.

[9] World Health Organization (WHO) \& United Nation Children Education Fund (UNICEF) WHO/UNICEF Joint Statement: Integrated Community Case Management (iCCM). Geneva/New York: WHO/UNICEF 2012.

[10] Aguayo, V.M., et al., Integrated program achieves good survival but moderate recovery rates among children with severe acute malnutrition in India. Am J Clin Nutr., 2013. 98(5): p. 1335-42.

[11] Muzigaba M, van Wyk B, and Puoane T., Management of severe acute malnutrition in children under 5 years through the lens of health care workers in two rural South African hospitals.; African Journal of Primary Health Care \& Family Medicine, 2018. 10 (1): p. 1547.

[12] Hobbs, B. and A. Bush, A 10 point plan for tackling acute malnutrition in under fives, in Acute malnutrition An everydays emergency2014, Generation Nutrition Campaign Report. p. 12-25.

[13] Munthali, T., et al., Mortality and morbidity patterns in under-five children with severe acute malnutrition (SAM) in Zambia: a five-year retrospective review of hospital-based records (2009 -2013). Archives of Public Health, 2015. 73(23).

[14] Kerac, M., et al., Follow-up of post-discharge growth and mortality after treatment for severe acute malnutrition (FuSAM study): a prospective cohort study. PLoS One, 2014. 9(6): p. 1-10.

[15] Kebede, S., Survival Status and Predictors of Mortality among Children Aged 0-59 Months with Severe Acute Malnutrition Admitted to Stabilization Center at Sekota Hospital Waghemra Zone. J Nutr Disorders Ther „, 2015. 5(2): p. 160-171.

[16] Melaku, G., M.B. Afework, and T. Mache, Treatment Outcomes and Associated Risk Factors of Severely Malnourished under Five Children Admitted to Therapeutic Feeding Centers of Mekelle City, Northern Ethiopia. Open Access Library Journal, 2014. 1(4): p. 1-9.

[17] Abdu Oumer, Firehiwot Mesfin, and Melake Demena, Survival Status and Predictors of Mortality among Children Aged 0-59 Months Admitted with Severe Acute Malnutrition in Dilchora Referral Hospital, Eastern Ethiopia. East African Journal of Health and Biomedical Sciences, 2016. 1(1): p. 13-22.

[18] Thurstans, S., The sphere project Humanitarian Charter and Minimum Standards in Humanitarian Response: Minimum Standards in Food Security and nutrition: Management of acute malnutrition and micronutrient deficiencies, The sphere project. 2011 p. 168-9.

[19] Abdullahi M, Houshiarrad A, Abtahi M, Esmaeli M, Pouraram H, Khoshfetrat MR, et.al. (2013). the nutrition knowledge level of physicians, nurses and nutritionists in some educational hospitals. Journal of Paramedical Sciences 4 (winter 2013) supplement ISSN 2008-4978.

[20] Gawde SR, B.S., Patel TC. Drug utilization pattern in pregnant women attending antenatal out Patient Department of a tertiary care hospital. British journal of pharmaceutical research, 2013; 3(1): 2231-2919.

[21] Tafese $\mathrm{Z}$ and Shele A, Knowledge, attitude and practice towards malnutrition among health care workers in Hawassa City, Southern Ethiopia. . Global Journal of Public Health Research, 2015. 1(1): p. 001-008.

[22] Victor Mogre, et al., Nurses' knowledge and attudes regarding malnutriton in children and its management in Ghana. Curatonis 2017. 40(1): p. a1618-1625.

[23] Nurdan Yalcin, et al., Nutrition Knowledge Level of Nurses. Health Science Journal, 2013. 7( 1): p. 99-108.

[24] [Ajay Gaur and Akanksha Bansal, Severe acute malnutrition: what is lacking in office practice. Int J Contemp Pediatr, 2016. 3(3): p. 1064-1070.

[25] Tefera, C., et al., Treatment Outcome and Associated Factors among Under-Five Children with Severe Acute Malnutrition Admitted to Therapeutic Feeding Unit in Woldia Hospital, North Ethiopia J Nutr Food Sci 2014. 4(6).

[26] [Moise Muzigaba, Brian van Wyk, and Thandi Puoan, Management of severe acute malnutriton in children under 5 years through the lens of health care workers in two rural South African hospitals. African Journal of Primary Health Care \& Family Medicine, 2018. 10(1).

[27] [De Onis, M. and Z. Weise Prinzo, Managing children with severe acute malnutrition--what's new? A health policy perspective. Indian Pediatr, 2014. 51(1): p. 17-8.

[28] [Puett C and Guerrero S, Barriers to access for severe acute malnutrition treatment services in Pakistan and Ethiopia: a comparative qualitative analysis. Public Health Nutr, 2015. 18: p. 1873-1882.

[29] World Health organization (WHO), WHA Global Nutrition Targets 2025: Anaemia Policy Brief, Nutrition, Editor 2011: Geneva. 


\section{BIOGRAPHY OF AUTHOR}

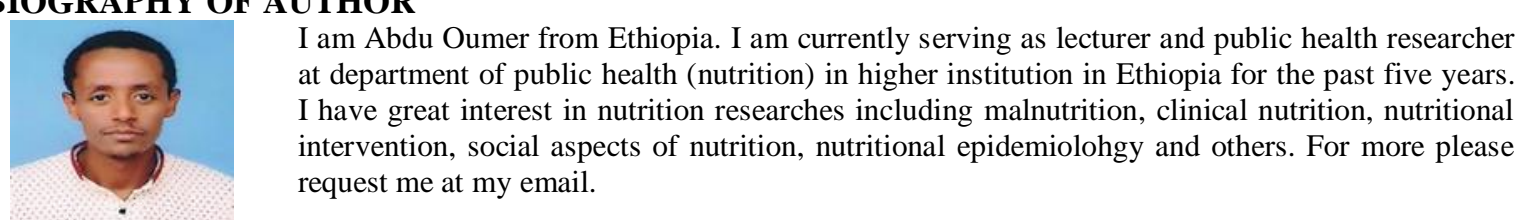

Int. J. Public Health Sci. Vol. 8, No. 2, June 2019: 219 - 228 